\title{
STELLAR ACTIVITY CYCLES
}

\author{
Dainis Dravins \\ Lund Observatory \\ Box 43 \\ S-22100 Lund \\ Sweden
}

ABSTRACT. The main features of solar and stellar activity cycles are summarized, followed by a discussion of some outstanding observational problems for the future.

\section{SOLAR ACTIVITY CYCLES}

1.1. What has been observed?

The classical indicators for the solar activity cycle are the sunspot number and the magnetic field polarity, which vary with the we11-known periods of 11 and 22 years, respectively. However, also many other parameters vary during the solar cycle, e.g.

$\begin{array}{ll}\text { Corona: } & \begin{array}{l}\text { Solar wind velocity } \\ \text { Coronal density structure } \\ \text { X-ray bright points }\end{array} \\ \text { Chromosphere: } & \begin{array}{l}\text { Ultraviolet and radio fluxes } \\ \text { Emission and absorption 1ines } \\ \text { Flare frequency }\end{array} \\ \text { Photosphere: } \quad \begin{array}{l}\text { Magnetic field patterns } \\ \text { Granulation structure } \\ \text { Spectral irradiance? }\end{array} \\ \text { Deeper 1ayers: Temperature gradient changes? } \\ \text { Torsional oscillations? } \\ \text { P-mode oscillation frequencies? }\end{array}$

The observational difficulties often increase as one goes to deeper layers in the Sun. Solar-cycle variations in the shape of the corona seen at total eclipses can be watched with the unaided eye; chromospheric activity changes can be followed with a modest telescope, but more powerful spectroscopic or imaging instrumentation is required to reveal 
photospheric changes and a convincing detection of variations in the solar interior may require great sophistication in the observations and in their interpretation. At the same time, the more fundamental mechanisms behind solar activity are likely to be found in the deeper 1ayers.

Although all the parameters 1 isted probably vary with an 11 or 22 year period, it is important to note that many of them are definitely not in phase with one another or with the sunspot number indicator.

As examples of indicators that seem to be completely out of phase, we note in the photosphere the number density of facular bright points (presumably associated with magnetic field concentrations), which have a minimum at sunspot number maximum (Muller and Roudier, 1984). In the chromosphere, the same seems to hold for the $D_{3}$ emission seen at total eclipses (Gulyaev, 1975), and in the corona for the density of X-ray bright points (Golub et al., 1979). Chromospheric Ca II H \& K emission and He I $\lambda 1083 \mathrm{~nm}$ absorption indicators are phaseshifted by some year relative to the sunspot number cycle. There exist further varying parameters related to sunspots besides their number, e.g. the umbral brightness. An overview of solar cycle properties is by Newkirk and Frazier (1982).

Perhaps one could compare the solar activity cycle to that of a blossoming apple garden. This garden will have its general 12-month activity cycle, but the activity maxima for the leaves, the flowers, and the fruits will definitely be out of phase with respect to one another. A challenge for an observer would be to understand the fundamenta1 12-month cycle from limited observations of the variation in the number of leaves, of the brightness of flowers or the flavor of the fruits.

\subsection{Outstanding Observational Problems}

A number of fundamental observational problems can be identified, for example:

1.2.1. Magnetic flux variation during the solar cycle? The amount of magnetic flux emerging through the solar photosphere is an awkward quantity to measure. The reason is that the spectral line polarization signal being measured by solar magnetographs depends in a complex manner upon the detailed surface distributions of magnetic flux densities, and their correlations with temperature inhomogeneities. Further, the apparent net flux in regions with intermingled polarities also very much depends on telescope resolution and atmospheric seeing. It has even been suggested that the solar cycle might be characterized as an oscillation in magnetic wavenumber space, with little variation in the average total amount of emerging flux (Golub et al., 1979).

1.2.2. Changes in the deeper layers and in the convection zone? Solar seismology has now measured p-mode oscillation eigenfrequencies during a sizable fraction of an 11-year cycle, and secular changes in these oscillations are suggested (Woodard and Noyes, 1986). One could perhaps suspect that some process in a magnetic dynamo would, during some phase of the cycle, lead to an increased amount of magnetic fluxtubes in the 
upper convection zone, and thus to an additional source of pressure support for the solar mantle, which should affect the oscillation frequencies being measured.

1.2.3. Activity in the ancient Sun and stability of the cycle period? Past activity of the Sun can be studied through proxy indicators such as the ${ }^{14} \mathrm{C}$ radiocarbon abundance in samples with known age. However, following its production by cosmic rays in the upper atmosphere, ${ }^{14} \mathrm{C}$ is stored in the atmosphere and in the oceans for some decade before being absorbed by e.g. a tree, and thus the 11 -year cycle is washed out in ${ }^{14} \mathrm{C}$ data. In principle, there exist other radionuclides such as ${ }^{10} \mathrm{Be}$ (half-life $\left.2 \cdot 10^{6} \mathrm{y}\right)$ that might allow one to follow the year-by-year activity variations back over millions of years (e.g. Pepin et a1., 1980).

Geological deposits of glacial sediments have been found that show an 11-year variation in the deposit thickness, and this variation has been suggested to be of solar origin, via a terrestrial climate response (Williams, 1981). If this suggestion of a solar origin is correct, this could be evidence for the stability of the 11 -year period over $\simeq 10^{9}$ years (although the activity Zevel may have varied considerably). To evaluate the plausibility of such climate modifications, it would be of great interest to measure the irradiance variations over at least the present 11- and 22-year cycles. Astronomical data have been searched for periods other than these, but the limited timespan of historical observations does not really lend support for the significance of other cycle periods. Some hint of the future sun might be obtained through studies of stars that are near solar-twins, only correspondingly older. Such an ongoing study of an old G2 subgiant (Dravins et a1., 1983) shows the activity level lower than solar, and an indication of variations that cannot exclude a cycle around 11 years.

\section{STELLAR ACTIVITY CYCLES}

\subsection{What has been observed?}

The current great interest for stellar activity cycles is to no small part due to Olin Wilson's extensive series of observations of Ca II K chromospheric fluxes from cool main-sequence stars (Wilson, 1978; Wilson et al., 1981). Evidence of cyclic activity now exists for several classes of stars:

2.1.1. Ordinary stars: chromospheric activity cycles. A large number of late-type main-sequence stars have now been monitored for some 20 years, and a fair number of activity cycles have been identified, the shortest of about 3 years. There seems to be no obvious correlation between the cycle period and other primary stellar parameters. What is being measured is the photometric flux in a narrow band around $\mathrm{Ca}$ II $\mathrm{K}$ line center, normalized to the stellar continuum. For the Sun, such an activity index mainly gives a measure of the active region plage area coverage and brightness. Besides the identification of activity changes over several years, the rotational modulation of the $C a$ II $K$ emission can be seen in 
several cases. This modulation must originate from stellar active regions rotating into and out from the visible stellar disk. A very intriguing suggestion of changes in this rotational period over some years has been seen, possibly a measure of the stellar differential rotation with latitude. In analogy with the solar case, the preferred latitude of stellar activity might be drifting with phase in the activity cycle. A well-written review of the results from these programs, carried out mainly at the Mt.Wilson Observatory, is by Baliunas and Vaughan (1985).

2.1.2. Spotted stars: irradiance cycles. Large and cool starspots are known to exist on some evolved and active stars in some binary systems, in particular those of the RS CVn and BY Dra types. From photographic plate collections, it has been possible to follow the brightness of some of these for almost a century, giving evidence to stellar irradiance cycles with periods up to perhaps 60 years (Hartmann et a1., 1981). In eclipsing binaries of these types, a photometric 'wave' can sometimes be seen when the starspots (marking stellar rotation) lead or lag the geometrical stellar eclipse. Such behavior seems consistent with differential rotation with latitude in spot cycles with deduced periods in the range 4 - 10 years. A discussion of spotted stars is included in the reviews by Paternò (1983) and Baliunas and Vaughan (1985).

Also more ordinary high-activity stars possess irradiance variations due to the apparent presence of dark spots that seem to correlate with bright chromospheric emission lines, indicating that starspots and stellar active regions are nearly cospatial (Blanco et al., 1979; Radick et al., 1983).

2.1.3. Flare stars: flare frequency cycles? The strong variability of many very late-type dwarfs, together with their photometric faintness, makes the monitoring of chromospheric emission lines rather difficult in searches for activity cycles. Pettersen et a1. (1986) instead collected a substantial amount of high-speed U-band photometry for the flare star $\mathrm{AD}$ Leo (dM4e) spanning 14 years. Supposing that the data are complete for all stronger flares, their frequency of occurence was studied as a function of time, and some (margina1?) evidence for an 8-year cycle found. Irrespective of the reality of the cycle in $A D$ Leo, these types of studies are very promising for the search of possible cyclic activity in very late-type stars which might be convective throughout. For the modeling of possible activity cycles in such stars, several of the standard magnetic dynamo theories will not be applicable.

\subsection{Outstanding Observational Problems}

2.2.1. Magnetic cycles in ordinary stars? Although the term 'magnetic' is sometimes loosely being used to describe stellar chromospheric activity, it should be remembered that (with the exception of periodic phenomena in Ap and other magnetic stars) no stellar magnetic cycles have yet been identified. Since the magnetic field is believed to be a fundamental parameter underlying most stellar activity, such observations would obviously be of considerable interest. One possible way could be to 
search for circular polarization reversals in stellar microwave radio bursts, as has been tried by Gibson (1984). If, in analogy with the Sun, the two polarity areas in stellar active regions are not symmetric, and one of them has a more spread-out magnetic field, the result must be that e.g. the gyroresonance opacity will make the radio source optically thick at different atmospheric heights above the two different polarities. Since the measured polarization depends on the ratio of effective emission areas for each magnetic polarity, reversals in a magnetic cycle should show up as circular polarization reversals in microwave emission. (This is essentially the inverse of the problem discussed in section 1.2.1. the discrepancy between a magnetic polarization signal and the true stellar magnetic flux.)

2.2.2. Cyclic activity in ordinary evolved stars? Most studies so far have been made for either main-sequence stars or for special evolved stars in close binary systems. Among the original main-sequence stars in Wilson's early survey, improved spectral data has now reclassified some as giants, for which there thus are long time-sequences available. There is an example of a G2 III star with an apparently cyclic behavior, not much different from other dwarf stars (Baliunas and Vaughan, 1985). The He I $\lambda 1083 \mathrm{~nm}$ absorption line in Capella (G5 III + G0 III) may vary on a timescale of $\simeq 10$ years (Kat sova and Shcherbakov, 1983), but in neither case the evolutionary state of the star is very well understood. It would obviously be of great interest for the understanding of the fundamental activity mechanisms, if the cyclic activity of a typical star could be followed along the stellar evolutionary path from the main sequence to the giant stage. Possibly, the (unfortunately faint) stars in some evolved galactic clusters could be suitable objects for such a study.

2.2.3. Starspot imaging throughout an activity cycle. One of the awaited breakthroughs in stellar astrophysics during the next decades is the direct imaging of stellar surfaces, enabled by the advent of very large telescopes and long-baseline interferometers. Such imaging in either the spectral continuum or in chromospheric emission lines should reveal the size, shape and location of active regions on the stellar disk. The monitoring of these with time should allow the study of their development and decay and reveal the presence (if any) of differential rotation or of latitude drifts of the activity zone. Awaiting such interferometers, spectroscopic methods allow a certain reconstruction of starspot images for at least spotted stars with moderately rapid rotation, as demonstrated in the Doppler imaging by e.g. Vogt and Penrod (1983). U1timately, stellar surface imaging (and spatially resolved stellar spectroscopy) will be required to gain a more realistic appraisal of stellar activity and stellar surface structure. Quite possibly, our present understanding of stellar surface features may be equally naive as was the understanding of planetary system objects before the revelation of their surfaces by spacecraft.

\section{ACKNOWLEDGEMENT}

This work was supported by the Swedish Natural Science Research Council. 


\section{REFERENCES}

Baliunas, S.L., Vaughan, A.H.:1985, Ann.Rev.Astron.Astrophys. 23, 379

Blanco, C., Catalano, S., Maril1i, E.:1979, Nature 280, 661

Dravins, D., Linde, P., Fredga, K., Gahm, G.:1983, BuZZ.AAS 15, 698

Gibson, D.M.:1984, in S.L.Baliunas, L.Hartmann, eds. Cool Stars, Stezlar Systems, and the Sun, Springer, p.197

Golub, L., Davis, J.M., Krieger, A.S.:1979, Astrophys.J. 229, L145

Gulyaev, R.A.:1975, Solar Phys. 44, 25

Hartmann, L., Bopp, B.W., Dussault, M., Noah, P.V., Klimke, A.:1981, Astrophys.J. 249, 662

Katsova, M.M., Shcherbakov, A.G.:1983, Astron.2h. 60, $267=$ Soviet Astr. 27, 153

Muller, R., Roudier, T.:1984, in The Hydromagnetics of the Sun, ESA $\mathrm{SP}-220, \mathrm{p} .51$

Newkirk, G., Frazier, K.:1982, Physies Today 35, No.4, 25

Paternō, L.:1983, in J.C.Pecker, Y.Uchida, eds. Active Phenomena in the Outer Atmosphere of the Sun and Stars, Paris, p.343

Pepin, R.0., Eddy, J.A., Merril1, R.B., eds.:1980, The Ancient Sun = Geochim. Cosmochim.Acta Suppi. 13, Pergamon

Pettersen, B.R., Panov, K.P., Sandmann, W.H., Ivanova, M.S.:1986, Astron. Astrophys., submitted

Radick, R.R., Wilkerson, M.S., Worden, S.P., Africano, J.L., Klimke, A., Ruden, S., Rogers, W., Armandroff, T.E., Giampapa, M.S.:1983, Publ. Astron. Soc. Pacific 95,300

Vogt, S.S., Penrod, G.D.:1983, Publ.Astron.Soc.Pacific 95, 565

Williams, G.E.:1981, Nature 291, 624

Wilson, O.C.:1978, Astrophys.J. 226,379

Wilson, O.C., Vaughan, A.H., Mihalas, D.:1981, Scient.American 244, No. 2,82

Woodard, M.F., Noyes, R.W.:1986, Nature, submitted 